\title{
NOTES ON WHITEHEAD SPACE OF AN ALGEBRA
}

\author{
M. ARIAN-NEJAD
}

Received 2 August 2001

Let $R$ be a ring, and denote by $[R, R]$ the group generated additively by the additive commutators of $R$. When $R_{n}=M_{n}(R)$ (the ring of $n \times n$ matrices over $R$ ), it is shown that $\left[R_{n}, R_{n}\right]$ is the kernel of the regular trace function modulo $[R, R]$. Then considering $R$ as a simple left Artinian $F$-central algebra which is algebraic over $F$ with $C h a r F=0$, it is shown that $R$ can decompose over $[R, R]$, as $R=F x+[R, R]$, for a fixed element $x \in R$. The space $R /[R, R]$ over $F$ is known as the Whitehead space of $R$. When $R$ is a semisimple central $F$ algebra, the dimension of its Whitehead space reveals the number of simple components of $R$. More precisely, we show that when $R$ is algebraic over $F$ and $\operatorname{Char} F=0$, then the number of simple components of $R$ is greater than or equal to $\operatorname{dim}_{F} R /[R, R]$, and when $R$ is finite dimensional over $F$ or is locally finite over $F$ in the case of $\operatorname{Char} F=0$, then the number of simple components of $R$ is equal to $\operatorname{dim}_{F} R /[R, R]$.

2000 Mathematics Subject Classification: 12E15, 16K40.

1. Introduction. Additive commutator elements of a ring $R$ and the groups and structures they make have a great role in the general specification of a ring, and their study is one of the approaches to recognize rings in noncommutative ring theory $[2,3,4,5]$. The reason is clear, they have covered the secrets of noncommutative behaviour of the structure. In recent years, these elements are returned once again under a full consideration, and a lot of wonderful works has been done on them $[1,10,11,12,13]$. Our study here is also among these studies, and it reveals some of bilateral relations between substructure given by additive commutators (the additive commutator group $[R, R]$, the additive Whitehead group, and the space $R /[R, R]$ ) and some characteristics of the ring. In what follows let $R$ be a ring. By $[R, R]$ we denote the group generated additively by the additive commutators of $R$. Following [2], the additive group $R /[R, R]$ is called the additive Whitehead group of $R$. This group is an $F$-vector space when $R$ is a central $F$-algebra, and is called the Whitehead space of $R$.

2. Results. Our first result is about the additive commutator subgroup of a matrix ring over a given ring.

Proposition 2.1. Let $R$ be a unitary ring and let $R_{n}=M_{n}(R)$ be the ring of $n \times n$ matrices over $R$. Consider the regular trace function on $R_{n}$, as $\operatorname{tr}: R_{n} \rightarrow R$, then

$$
\left[R_{n}, R_{n}\right]=\left\{A \in R_{n} \mid \operatorname{tr}(A) \in[R, R]\right\} .
$$

Proof. The inclusion " $\subseteq$ " follows by the fact that $\operatorname{tr}(A B-B A) \in[R, R]$. In order to show the reverse inclusion, let $\left\{E_{i j}\right\}$ be the matrix units and note that if $i \neq j$, we have $E_{i j}=E_{i i} E_{i j}-E_{i j} E_{i i} \in\left[R_{n}, R_{n}\right]$ and $E_{i i}-E_{j j}=E_{i j} E_{j i}-E_{j i} E_{i j} \in\left[R_{n}, R_{n}\right]$. For any 
$A=\left(a_{i j}\right) \in R_{n}$, we have the following congruence:

$$
A=\Sigma a_{i j} E_{i j} \equiv \Sigma a_{i i} E_{i i} \equiv \Sigma a_{i i} E_{11}\left(\bmod \left[R_{n}, R_{n}\right]\right) .
$$

In particular, if $\operatorname{tr}(A) \in[R, R]$, then $A \in\left[R_{n}, R_{n}\right]$.

COROLLARY 2.2. Consider the trace function on $R_{n}$ module of $[R, R]$. Clearly the group isomorphism $R_{n} /\left[R_{n}, R_{n}\right] \cong R /[R, R]$ can be derived.

THEOREM 2.3. Let $R$ be a left Artinian central simple F-algebra which is algebraic over $F$ with Char $F=0$. Then $R$ decomposes over $[R, R]$ as $R=F x+[R, R]$, for a fixed $x \in R$.

Proof. By Wedderburn-Artin theorem, $R=M_{n}(D)$ for a division ring $D$ and suitable $n \in \mathbb{N}[6,14]$. We divide our proof into two parts.

(i) Let $n=1$, in other words let $R=M_{1}(D)=D$ be a division ring. Let $a \in R$ and let $f(t)=t^{r}+b_{1} t^{r-1}+\cdots+b_{r}$ be the minimal polynomial of $a$ over $F$, where $b_{i} \in F$, $i=1,2, \ldots, r$ and $r=\operatorname{dim}_{F} F(a)$. By the Wedderburn theorem [9, page 265], $f(t)$ splits completely in $R[t]$, this means that there exists $c_{i} \in R^{*}=D-\{0\}, i=1,2, \ldots, r-1$, such that $f(t)=(t-a)\left(t-c_{1} a c_{1}^{-1}\right) \cdots\left(t-c_{r-1} a c_{r-1}^{-1}\right)$. Then we have

$$
\begin{aligned}
\operatorname{Tr}_{F(a) / F}(a) & =a+c_{1} a c_{1}^{-1}+c_{2} a c_{2}^{-1}+\cdots+c_{r-1} a c_{r-1}^{-1} \\
& =r a+\left(c_{1} a c_{1}^{-1}-a\right)+\cdots+\left(c_{r-1} a c_{r-1}^{-1}-a\right) \\
& =r a+\left(c_{1}\left(a c_{1}^{-1}\right)-\left(a c_{1}^{-1}\right) c_{1}\right)+\cdots+\left(c_{r-1}\left(a c_{r-1}^{-1}\right)-\left(a c_{r-1}^{-1}\right) c_{r-1}\right) \\
& =r a+d_{1}+d_{2}+\cdots+d_{r-1}=r a+d
\end{aligned}
$$

where $d_{1}, \ldots, d_{r-1}, d \in[R, R]$. This simply yields $a \in F+[R, R]$ which imply that $R=$ $F+[R, R], x=1$.

(ii) Let $n \in \mathbb{N}$ be an arbitrary positive integer. We have $R=M_{n}(D)$, where $D$ is a division ring. By (i), $D=F+[D, D]$, so

$$
R=M_{n}(D)=M_{n}(F+[D, D])=M_{n}(F)+M_{n}([D, D]) \subseteq M_{n}(F)+[R, R] \subseteq R .
$$

This implies that $R=M_{n}(F)+[R, R]$. By this formula, given $A \in R$, there exist $B \in$ $M_{n}(F)$ and $C \in[R, R]$ such that $A=B+C$, hence $A=(B-(\operatorname{tr} B / n) I)+(\operatorname{tr} B / n) I+C$, where $I$ is the identity matrix of size $n$. By Proposition 2.1, $(B-(\operatorname{tr} B / n) I) \in[R, R]$, and $A=(\operatorname{tr} B / n) I+((B-(\operatorname{tr} / n) I)+C)$, consequently

$$
R=F I+[R, R], \quad x=I
$$

To see a different statements and initial ideas of these theorems we refer the reader to [1, 2]. Also a multiplicative version of Theorem 2.3 could be found in [11].

Now, we are going to state our main result, which is about the Whitehead space of a semisimple ring. This theorem is a generalization of a nice theorem due to $R$. Brauer [8, page 130]. 
THEOREM 2.4. Let $R$ be a left Artinian semisimple central $F$-algebra and let $k$ be the number of left simple components of $R$. Then,

(i) if $R$ is algebraic over $F$ and Char $F=0$, then $k \geq \operatorname{dim}_{F} R /[R, R]$;

(ii) if $R$ is finite dimensional over $F$, or is locally finite over $F$, and Char $F=0$, then $k=\operatorname{dim}_{F} R /[R, R]$.

Proof. Consider the following chain of functions:

$$
R \stackrel{f_{1}}{\longrightarrow} M_{n_{1}}\left(D_{1}\right) \times \cdots \times M_{n_{k}}\left(D_{k}\right) \stackrel{f_{2}}{\longrightarrow} D_{1} /\left[D_{1}, D_{1}\right] \times \cdots \times D_{k} /\left[D_{k}, D_{k}\right],
$$

where $f_{1}$ is the isomorphism given by the Wedderburn-Artin theorem for the decomposition of a semisimple left Artinian ring into a direct product of simple ring [6, 14], and $f_{2}$ is the $F$-algebra homomorphisms, by considering component-wise the trace function on $M_{n_{i}}\left(D_{i}\right) \bmod \left[D_{i}, D_{i}\right], i=1, \ldots, k$.

By Proposition 2.1 we have, $\operatorname{ker}\left(f_{2} \circ f_{1}\right)=[R, R]$, noting that $[R, R] \cong\left[R_{1}, R_{1}\right] \times \cdots \times$ $\left[R_{k}, R_{k}\right]$, where $R_{n_{i}}=M_{n_{i}}\left(D_{i}\right), i=1, \ldots, k$. Therefore the following $F$-isomorphism holds:

$$
R /[R, R] \cong D_{1} /\left[D_{1}, D_{1}\right] \times \cdots \times D_{k} /\left[D_{k}, D_{k}\right]
$$

It remains to compute the dimension of Whitehead space of a division ring in the two cases (i) and (ii) above.

First let $D$ be algebraic over $F$ and $\operatorname{Char} F=0$. We show that any two elements $\bar{a}, \bar{b} \in D /[D, D]$ are linearly dependent. By Theorem 2.3, there exist elements $\alpha, \beta \in F$ and $d_{1}, d_{2} \in[D, D]$, such that $a=\alpha+d_{1}$ and $b=\beta+d_{2}$. In other words, $\beta \bar{a}-\alpha \bar{b}=\overline{0}$ in $D /[D, D]$. Hence in this case $\operatorname{dim}_{F} D /[D, D] \leq 1$.

Now let $D$ be finite dimensional $F$-central algebra. Let $\mathrm{RT}_{D / F}: D \rightarrow F$ be the reduced trace function which is surjective by [7, page 148]. Furthermore, by a theorem of Amitsur and Rowen [5, page 171] its kernel is equal to $[D, D]$ and so it is a hyperplane over $F$, in this case $\operatorname{dim}_{F} D /[D, D]=1$.

As a latter case let $D$ be a locally finite division ring over it's center $F$ and Char $F=0$. Now consider the function TR: $D \rightarrow F$ defined by

$$
\operatorname{TR}(x)=\frac{1}{\operatorname{deg}_{F}(x)} \operatorname{Tr}_{F(x) / F}(x)
$$

we show that this function is an $F$-linear surjective map, whose kernel is $[D, D]$. The claim then is clear.

First note that in this case $1 \notin[D, D]$, for if $1 \in[D, D]$, then there exist some $x_{i}$ 's and $y_{i}$ 's in $D$, such that $1=\sum\left(x_{i} y_{i}-y_{i} x_{i}\right)$. Let $D_{1}$ be the division ring generated by $F$ together with $x_{i}$ 's and $y_{i}$ 's. Taking the reduced trace of $D_{1}$ over its centre of both sides of $1=\sum\left(x_{i} y_{i}-y_{i} x_{i}\right)$, we get a contradicting result. Therefore $[D, D] \cap F=\{0\}$. Now, by considering the trace formula (given in the proof of Theorem 2.3) for elements $a$, $b$ and $\lambda a+b(\lambda \in F)$ in $D$, it is readily verified that

$$
\frac{1}{r} \operatorname{Tr}(\lambda a+b)=\frac{\lambda}{n} \operatorname{Tr}(a)+\frac{1}{m} \operatorname{Tr}(b),
$$


where $r, n$, and $m$ are degrees of $\lambda a+b, a$ and $b$. So TR is $F$-linear. The surjectivity is clear. In order to specify the kernel of TR, consider the trace formula for elements of $[D, D]$. Suppose that $a \in[D, D]$. Now, we have $\operatorname{Tr}_{F(a) / F}(a)=n a+d \in[D, D] \cap F$, where $n$ is the degree of $a$ over $F$ and $d \in[D, D]$. Therefore $\operatorname{TR}(a)=0$. By the same argument we can see that if $\operatorname{TR}(a)=0$, then $a \in[D, D]$.

ACKNOWLEDGMent. The author is indebted in part to the Research Council of the University of Zanjan for support.

\section{REFERENCES}

[1] S. Akbari and M. Arian-Nejad, Left Artinian algebraic algebras, Algebra Colloq. 8 (2001), no. 4, 463-470.

[2] S. Akbari, M. Arian-Nejad, and M. L. Mehrabadi, On additive commutator groups in division rings, Results Math. 33 (1998), no. 1-2, 9-21.

[3] S. Akbari and M. Mahdavi-Hezavehi, Normal subgroups of $\mathrm{GL}_{n}(D)$ are not finitely generated, Proc. Amer. Math. Soc. 128 (2000), no. 6, 1627-1632.

[4] S. Akbari, M. Mahdavi-Hezavehi, and M. G. Mahmudi, Maximal subgroups of $\mathrm{GL}_{1}(D)$, J. Algebra 217 (1999), no. 2, 422-433.

[5] S. A. Amitsur and L. H. Rowen, Elements of reduced trace 0, Israel J. Math. 87 (1994), no. 1-3, 161-179.

[6] P. M. Cohn, Algebra. Vol. 3, 2nd ed., John Wiley \& Sons, Chichester, 1991.

[7] P. K. Draxl, Skew Fields, London Mathematical Society Lecture Note Series, vol. 81, Cambridge University Press, Cambridge, 1983.

[8] I. N. Herstein, Noncommutative Rings, The Carus Mathematical Monographs, vol. 15, The Mathematical Association of America, Washington, DC, 1971.

[9] T. Y. Lam, A First Course in Noncommutative Rings, Graduate Texts in Mathematics, vol. 131, Springer-Verlag, New York, 1991.

[10] M. Mahdavi-Hezavehi, Extension of valuations on derived groups of division rings, Comm. Algebra 23 (1995), no. 3, 913-926.

[11]__ Commutator subgroups of finite dimensional division algebras, Rev. Roumaine Math. Pures Appl. 43 (1998), no. 9-10, 853-867.

[12] M. Mahdavi-Hezavehi and S. Akbari, Some special subgroups of $\mathrm{GL}_{n}(D)$, Algebra Colloq. 5 (1998), no. 4, 361-370.

[13] M. Mahdavi-Hezavehi, S. Akbari-Feyzaabaadi, M. Mehraabaadi, and H. Hajie-Abolhassan, On derived groups of division rings. II, Comm. Algebra 23 (1995), no. 8, 2881-2887.

[14] L. H. Rowen, Ring Theory, Academic Press, Massachusetts, 1991, Student edition.

M. ARIAN-NeJAD: DePARTMENT OF MATHEMATICS, UNIVERSity OF ZANJAN, ZANJAN, IRAN

E-mail address: arian@mai 1.znu.ac. ir 


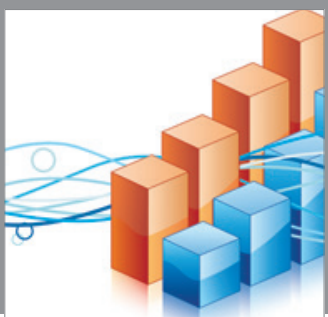

Advances in

Operations Research

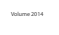

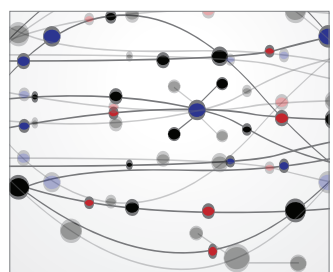

\section{The Scientific} World Journal
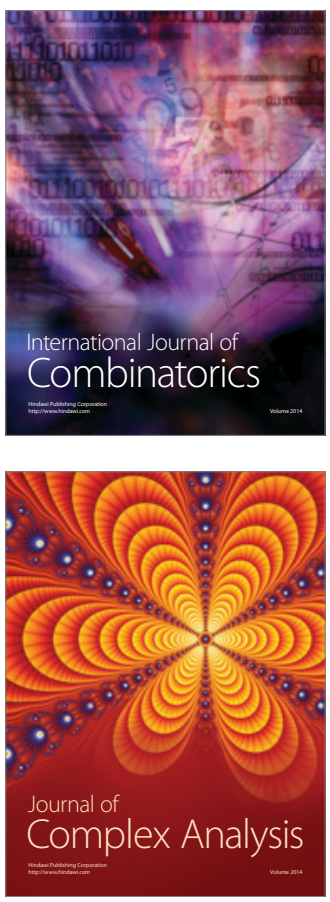

International Journal of

Mathematics and

Mathematical

Sciences
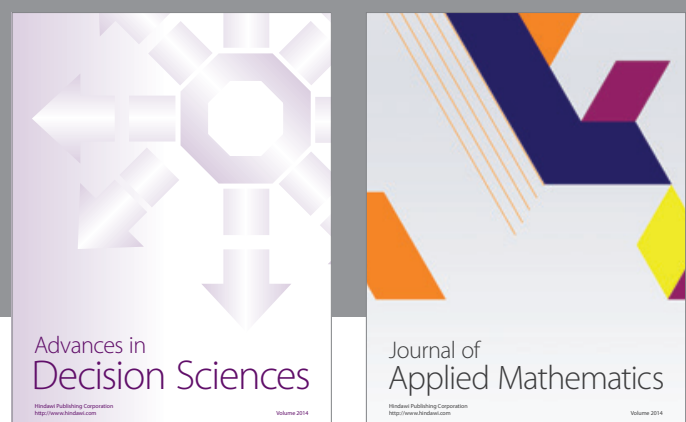

Journal of

Applied Mathematics
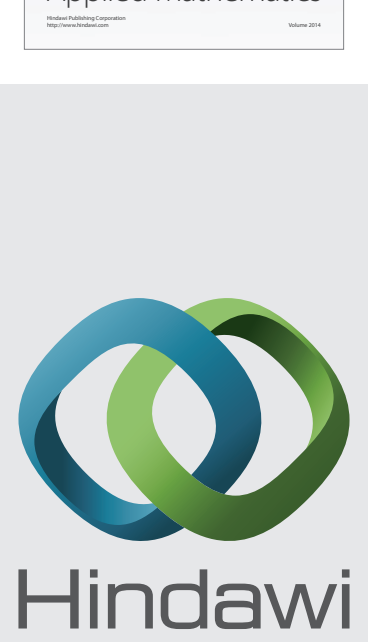

Submit your manuscripts at http://www.hindawi.com
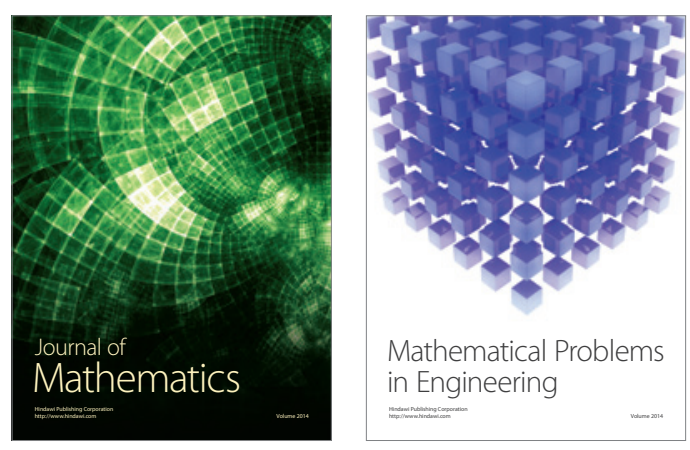

Mathematical Problems in Engineering
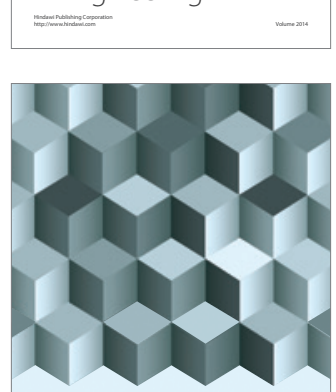

Journal of

Function Spaces
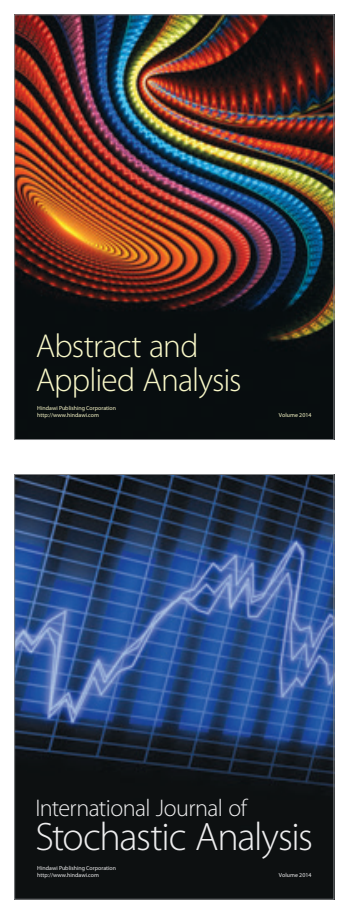

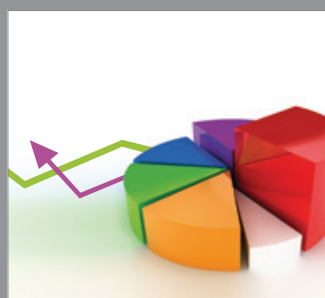

ournal of

Probability and Statistics

Promensencen
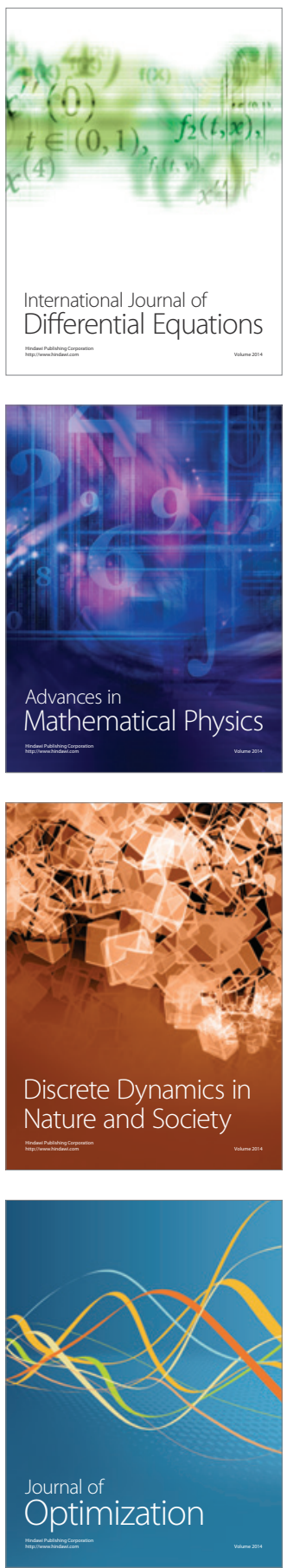